\title{
mebipred: identifying metal-binding potential in protein sequence.
}

\section{Aptekmann $\mathrm{AA}^{1,2^{\star}}$, Buongiorno $\mathrm{J}^{3}$, Giovannelli $\mathrm{D}^{2,4,5}$, Glamoclija $\mathrm{M}^{6}$, Ferreiro $\mathrm{DU}^{7}$, Bromberg $\mathrm{Y}^{1, *}$}

41 Department of Biochemistry and Microbiology, Rutgers University, 76 Lipman Dr, New Brunswick, 5 NJ 08873, USA

62 Institute of Marine and Coastal Sciences, Rutgers University, New Brunswick, NJ 08901, USA

73 Maryville College, Maryville, Tennessee, USA

84 Department of Biology, University of Naples Federico II, Naples, Italy

95 Institute for Marine Biological Resources and Biotechnology - IRBIM, National Research Council of

loltaly, CNR, Ancona, Italy

16 Department of Earth and Environmental Sciences, Rutgers University, New Brunswick, NJ 07102, 12 USA

137 Protein Physiology Lab, Departamento de Quimica Biologica, Facultad de Ciencias Exactas y ${ }_{14}$ Naturales, Universidad de Buenos Aires-CONICET-IQUIBICEN, Buenos Aires, 1428, Argentina

6* Corresponding author: arielaptekmann@bromberglab.org yanab@bromberglab.org,

Running Title: mebipred: Identifying metal-binding abilities from protein sequence.

Document statistics: Abstract $=233$, Text $=6590$ words, 135 references, 5 figures; 4 tables

Journal:

Journal name (current submission)

Submitted:

Abstract

${ }_{3}$ Metal-binding proteins have a central role in maintaining life processes. Nearly one-third of 24 known protein structures contain metal ions that are used for a variety of needs, such as 25catalysis, DNA/RNA binding, protein structure stability, etc. Identifying metal-binding ${ }_{26}$ proteins is thus crucial for understanding the mechanisms of cellular activity. However, 27experimental annotation of protein metal-binding potential is severely lacking, while 28computational techniques are often imprecise and of limited applicability.

${ }_{29}$ We developed a novel machine learning-based method, mebipred, for identifying metal3obinding proteins from sequence-derived features. This method is nearly $90 \%$ accurate in 3 recognizing proteins that bind metal ions and ion containing ligands. Moreover, the identity of 32ten ubiquitously present metal ions and ion-containing ligands can be annotated. mebipred is 33 reference-free, i.e. no sequence alignments are involved, and outperforms other prediction 34 methods, both in speed and accuracy. mebipred can also identify protein metal-binding 35 capabilities from short sequence stretches and, thus, may be useful for the annotation of 36 metagenomic samples metal requirements inferred from translated sequencing reads. We 37performed an analysis of microbiome data and found that ocean, hot spring sediments and soil 
microbiomes use a more diverse set of metals than human host-related ones. For humanhosted microbiomes, physiological conditions explain the observed metal preferences. ${ }_{3}$ Similarly, subtle changes in ocean sample ion concentration affect the abundance of relevant 4metal-binding proteins. These results are highlight mebipred's utility in analyzing smicrobiome metal requirements.

smebipred is available as a web server at services.bromberglab.org/mebipred and as a standalone package at https://pypi.org/project/mymetal/

10Keywords: Metal binding proteins; metagenome annotation; machine learning; 11 human microbiome; ocean microbiome; neural networks;

${ }_{12}$ Abbreviations used: mebipred, Metal-binding predictor; PDB, Protein Data Bank; 13BLAST, Basic local alignment search tool 


\section{Introduction}

${ }_{2}$ Proteins bind a diverse set of metal ion-containing cofactors and sustain the sfunctional requirements of life. Metal ions, e.g. iron, magnesium, copper, etc., and umetal-containing ligands, e.g. heme and iron-sulfur clusters, participate in protein sfolding/stability [1], DNA replication [2-4], catalysis [5], redox chemistry [5] and many other cellular activities. Proteins could thus be described as sophisticated electron transfer nanomachines that depend on transition metal ions to perform their functions 8[6-8]. Of the proteins whose three-dimensional structure is available in the Protein DataBank (PDB) [9], roughly a third $(49,996$ of 152,346$)$ are metal-binding proteins, a Iofinding which may be somehow related to their high abundance in nature. However, only a small fraction of metal-binding protein sequences has been identified overall. ${ }_{12}$ The Swiss-Prot [10] database, for example, contains over half a million $(564,638)$ smanually-curated protein sequences, of which $\sim 14 \%(94,720)$ are annotated as 14metal-binding; the binding activity of only a few of these $(<1 \%, 4,251$ proteins) has isthus far been experimentally verified (Feb 2020). Furthermore, of the nearly 180 ${ }_{16}$ million proteins in TrEMBL, which are generated via translation of sequenced 1 genome open reading frames and have no experimental annotations, only about five smillion sequences, i.e. less than $3 \%$, are annotated as metal-binding [11].

${ }_{9}$ Different levels of sequence redundancy in distinct databases may be an underlying zocause for this discrepancy. However, another major reason is that we are still unable 2 to accurately identify metal-binding proteins directly from their sequences and, in 22 some cases, even from their high resolution structures [12]. Experiments, e.g. mass 23spectrometry [13] and crystallography [14], can detect protein-metal interactions, but these analyses are expensive and time-consuming, as well as error-prone for both stechnical and biological reasons. For example, cambialistic proteins can use metal ${ }_{26}$ cofactors interchangeably [15] and thus are likely to be misclassified when experimentally assessed for binding of specific metals. Similarly, some experiments zsinclude the use of non-native metals for technical and/or crystallization purposes [16], 29the loss of metal ion-binding ability/specificity in the process of protein purification ${ }_{30}[17]$, or even simply incorrect identification of the bound metals due to low 
experimental resolution [18]. Thus, metal-binding annotation for most protein sequences is lacking and, likely, only a small portion of extant metal-binding protein sequences has been identified.

${ }_{4}$ There is no simple way to establish from sequence whether a protein binds a metal sor not, but there have been multiple attempts to predict binding of single ion ligands 6(including metals) from protein structures. While a complete account of all relevant 7methods present in the literature is beyond the scope of this work (for a review see 8 Lavechia et al [19]), here we highlight some trends in tool development.

9Metal-binding sites in proteins frequently comprise a shell of hydrophilic residues that locan be identified in the protein 3D structure [20]. For example, one algorithm [21] detects $\mathrm{Ca}^{2+}$ binding via identification of $\mathrm{Ca}^{2+}$ ion coordination by a layer of oxygen 12atoms supported by an outer shell of carbon atoms. Available structure-based methods use knowledge of hydrophilic shell residues to make predictions [20-24]. ${ }_{4}$ The main disadvantage of these approaches is that many such hydrophilic shells do 15not bind metals [25]. Additionally, structure-based methods are limited by the 16 relatively small number of available protein structures (157,668; PDB, Feb 2020) [9]. ${ }_{7}$ However, when a protein structure is available, these methods often attain better isperformance than ones based on sequence alone.

19To circumvent the limitation in the number of 3D structures, methods using homology 20 modeling of proteins were developed. Early attempts at this type of prediction (e.g. MetSite [26]) had poor performance (58\% precision at $28 \%$ recall). Overall, methods based on homology modeling tend to perform poorly when predicting sequences modeled with structural templates of less than $40 \%$ sequence identity; e.g. $42 \%$ 24 precision at $65 \%$ recall [27]. Moreover, these methods attain a better performance ${ }_{25}$ when focusing on a single metal ion than when trying to describe binding of multiple 26 ions, e.g. Liu et al calcium-binding site predictor (99\% precision at $75 \%$ recall) [28] 27 and Zhao et al zinc-binding predictor ( $90 \%$ precision at $72 \%$ recall) [29].

28The computational prediction of metal-binding can be similar in essence to the ${ }_{29}$ prediction of other functional characteristics of proteins from sequence, e.g. mutation 
leffects [30-32], residue importance [33], or subcellular localization [34]. Here, evolutionary profiles, predicted structure, physicochemical properties, and sequence ¿descriptors are combined as features for machine learning. One such approach to the prediction of metal-binding [35] has attained fairly high accuracy (70\% overall saccuracy). Other methods combine structural and sequence features in a process kknown as "fragment transformation" [23, 35, 36]; e.g. Lin et al [23] report accuracies 7above 92\%. Combining sequence, structure, and residue contact features in a srandom forest framework, the tool MetalExplorer [37] predicts the binding of eight metal ions. Performance across ions is varied, with a precision of $60 \%$ for recalls 10 ranging from $59 \%$ to $88 \%$.

11There are also structure-independent (purely sequence-based) methods to predict 2 metal binding. Function transfer by homology, i.e. the assumption that similar sequences perform similar functions, is one of the simplest ways to infer metal abinding for protein sequences. Similarity is often established by alignment methods. ${ }_{5}$ However, it remains unclear that there is a well-defined alignment score cutoff for 16identifying functionally similar proteins [38]. Moreover, sequence similarity, or even 17well-characterized homology, may be misleading as homologs may evolve to bind isdifferent metals due to changing environmental pressures [39]. It is also possible to predict metal binding using sequence conservation of residues near those directly 2ointeracting with $\mathrm{Zn}^{2+}, \mathrm{Cu}^{2+}, \mathrm{Fe}^{2+}, \mathrm{Fe}^{3+}$, and $\mathrm{Co}^{2+}$ ions with a high accuracy [40]; proteins binding other ions were not identified using this method. Pattern recognition 22 (e.g. Hidden Markov Models, HMMs, [41] and regular expressions, e.g. [42]) can also 23be used to expand the suspected set of metal binding sequences on the basis of 24remote homology. Unfortunately HMMs, designed to identify evolutionary conserved 25 sequence patterns, are too specific and, thus, not well-suited for de novo metal 26binding prediction.

More complicated sequence-based metal-binding predictors often use machine 28learning techniques (e.g. neural networks [43] support vector machines (SVM) [44, 2945], and random forests [46]). The performance these methods varies; e.g. Lin et al ${ }_{30}$ [47] reported high precision for all ions, albeit at recall as low as $35 \%$. Combining 
different methods to identify specific residues involved in metal binding, e.g. Znzbinding cysteines and histidines, produced high accuracy [45, 48-50]. Note that while sall of the above methods report good performance, we were unable to validate these reports using our own data as the webserver/standalone versions (where applicable) swere nonfunctional and downloadable scripts absent.

${ }_{6}$ Here we present mebipred (metal-binding predictor), a computational method for the prediction of protein metal binding potential based on sequence information alone. \&Our method is widely applicable because it doesn't depend on the existence of a high resolution structure, has a better performance (average precision/recall of $95 / 78 \%$ at 1odefault cutoff) and is faster (17,000 sequences/minute) than existing sequence-based itools, and can be used to predict metal binding using whole protein sequences as 12 well as short peptide fragments. The latter ability makes it potentially suitable for sannotation of shotgun-sequenced unassembled metagenomic data/reads. mebipred 14is also alignment-free and thus useful for the analysis of newly identified proteins 15 (with no known homologs). Finally, as mentioned previously, mebipred is the only ${ }_{16}$ currently publicly available method for sequence-based prediction of metal binding. 


\section{Methods}

${ }_{2}$ Datasets. We explored proteins binding $\mathrm{Na}, \mathrm{K}, \mathrm{Ca}, \mathrm{Mg}, \mathrm{Mn}, \mathrm{Fe}, \mathrm{Cu}, \mathrm{Ni}$, and $\mathrm{Zn}$ smetal-containing ligands, regardless of their oxidation state (e.g. Fe2+ and $\mathrm{Fe} 3+$ are uboth in the Fe class) or context (e.g. Fe-containing hemes are in the same class as ${ }_{5} \mathrm{Fe}$ ions). We retrieved all protein structures with these metal-containing ligands from the PDB (July 2019) and parsed them using the BioPython PDB module [51] 7(Supplementary Table 1). One naive approach to identify a set of metal-binding sproteins is to compile all structures that have a metal ion. However, in the case of heteromers, i.e. protein complexes that contain multiple nonidentical chains, it is topossible that only one of the chains binds the metal. We thus considered as metalbinding only the amino acid sequences/chains with at least one heavy atom within $5 \AA$ of the metal ion (METAL set). All other chains were included in the NO_METAL set, salong with all PDB structures that contained no metals at all. Note that this criterion for the differentiation of metal-binding/nonbinding chains could lead to disagreement is with existing metal-binding annotations.

GFeature extraction. To describe the proteins in our METAL and NO_METAL sets, ${ }_{17}$ we used only sequence-based features: 1) amino acid composition, 2) amino acid sphysicochemical properties, and 3 ) a count of the metal-binding amino acid 5 mers.

191. Amino acid composition: for each of the 20 standard amino acids, the percentage of the type of amino acid in the entire sequence. Total: 20 features.

2. Physicochemical properties of amino acids: We used a set of nine amino acid properties as described in Li et al. [52]: hydrophobicity, hydrophilicity, number of hydrogen bonds, volume, polarity, polarizability, solvent accessible surface area, net charge index of side chains, and average amino acid mass. For each of the properties (Fig. 1), we clustered sequence residues into three categories, Low (L), Medium $(\mathrm{M})$, and High $(\mathrm{H})$, using "Jenks natural breaks" criterion [53]; this measure seeks to minimize each class's average deviation from the class mean while maximizing each class's deviation from the means of the other classes. For each category, we further calculated its composition (C), i.e. the fraction of amino 
acids in the sequence belonging to the category; transitions (T), i.e. for every pair of sequential amino acids, the number of transitions from one category to another divided by sequence length; and distribution (D), i.e. the sequence position of the amino acid corresponding to category's first occurrence, $25 \%$ of occurrences, $50 \%, 75 \%$, and last occurrence, divided by sequence length. Total: 219 features.

$$
<<\text { FIG 1 >>> }
$$

73. For each protein structure in the PDB, individually for every metal, we identified all five amino acid-long subsequences (5mers) within $5 \AA$ of the bound metal ion (Fig. 2 ; i.e. for heme this means within $5 \AA$ of the Fe atom) and counted the frequency of their occurrence in all complete PDB sequences. Every query sequence is then decomposed into 5mers (via sliding window of 1) and the $5 \mathrm{mer}$ feature is computed as the sum of all occurrences of the individual metal-specific 5 mers in the PDB dictionary. We also included as a feature the sum of the frequencies across all ions. Total: 11 features.

\section{$<<$ FIG 2>>>}

Machine Learning. Using the above features, we trained a feed-forward Multi-Layer ${ }_{7}$ Perceptron (MLP) with back-propagation (BP) using the Keras [54] implementation in 18the machine learning framework Tensorflow [55]. Our model is a sequential network 19with the RMSprop [56] optimizer and a learning rate $(\operatorname{lr})=0.000005$. The optimization 20 of the learning rate parameter was done via gradient descent, i.e. starting with an ${ }_{21} \mid r=0.5$ we reduced it by an order of magnitude in each iteration of training and set the 22 value to the one that minimizes the loss (calculated as binary cross-entropy). All 3other parameters were set at default values according to the Keras manual [57]. The 24input layer consists of 219 nodes - one node per feature. There are two hidden 25layers, as these are sufficient to approximate most partition problems and require considerably less computational power than more hidden layers [58]. Each layer had 27219 nodes with a rectified linear unit activation function (or "Relu") and a dropout of 280.2. Finally, there is single node output layer, using the sigmoid activation function 29and a default prediction (yes/no) cutoff set at 0.5 . 
We trained and tested our model for identifying metal-binding proteins using ten-fold cross-validation as follows: (1) we clustered sequences at 70\% identity using CD-HIT [59] and used the representative sequences of each cluster for training; (2) we split the resulting sequences into positives (metal binding) and negatives (nonbinding), sand further divided each set into ten equally populated groups; (3) we built ten models by rotating through the ten splits using one positive and one negative group for testing and training with the other nine positive and negative groups. Since snegatives in our set are more frequent than positives, we balanced the sets by randomly down-sampling the negatives to the same number as the positives for each comodel training. Note that the ten models were used to estimate the performance of the method, while the final mebipred model was constructed using all sequences and established parameters.

${ }_{3}$ We additionally trained individual models with the same set of features to predict the abinding of specific metals. We used the same modeling procedure and parameters sas described above, only adding one more feature -- the score of the general metal16binding model above.

Performance metrics. To measure the performance of our method we calculated lsoverall accuracy, as well as positive precision, recall, and F-measure (all in Eqn. 1). ${ }_{19}$ True positives (TP) are metal-binding proteins predicted as metal-binding, false 20positives (FP) are metal non-binding proteins predicted as metal binding, false 21 negatives (FN) are metal-binding proteins predicted as metal non-binding, and true 2 negatives (TN) are metal non-binding proteins predicted metal non-binding.

$$
\begin{gathered}
\text { Precision }=\frac{\text { True Positive }}{\text { True Positive }+ \text { False Positive }} \quad \text { Recall }=\frac{\text { True Positive }}{\text { True Positive }+ \text { False Negative }} \\
F=2 X \frac{\text { Precision } X \text { Recall }}{\text { Precision }+ \text { Recall }} \quad \text { Accuracy }=\frac{\text { True Positive }+ \text { True Negative }}{\text { Total predictions }} \quad \text { (Eqn. 1) }
\end{gathered}
$$

${ }_{5}$ Comparing model performance to existing tools. To compare our method to a simple 26alignment-based approach, we extracted all sequences from the PDB. We generated 27 a database of these sequences using the makeblastdb (-blastdb_version=5 and no extra parameters). We then ran BLAST (ncbi-blast+ V. 2.10.4) [60,61] with default 
parameters (evalue 1; max_target_seqs 1000000) for all-to-all comparisons of 2protein sequences in this database. We used as gold standard our METAL and ${ }_{3}$ NO_METAL sets. For each e-value threshold, we counted the number of TP, FP, TN, and FN. Since we wanted to evaluate the use case where an unknown sequence is sbeing annotated, we excluded self-hits from BLAST results but did not exclude hits to homologous sequences.

${ }_{7}$ We further compared our performance to that of multiple published tools. For MetalDetector2 (Table 2), we used a set of non-redundant metal binding PDB structures described as the evaluation set of that method's manuscript [50] (extracted 1oin 2011). We also compared mebipred to two sequence based methods [40,46] and 11a structure based method (MIB) [23] using the data from the BioLip database [62] ${ }_{12}$ (non-redundant at $90 \%$ sequence identity; Table 3 ).

${ }_{3}$ Generating short peptides. We took all 50-residue or longer protein sequences in the PDB (445,763 protein sequences) and, using a sliding window of 1 , cut them into ${ }_{15}$ fragments of 50 residues (101,054,024 fragments).

6 Metagenomic sample processing. To analyse metagenomic samples, reads were 17trimmed with trimmomatic [63] using default parameters. Trimmed reads were filtered 18with phred [64] using a score cut-off of 28 . Reads were then analysed in two ways:

1. All reads where translated into the six possible reading frames using the standard bacterial codon table from Biopython [65]. Translated reads containing less than 15 amino acids where discarded. Remaining reads were used as input to mebipred

2.Reads were assembled using metaSPAdes [66] with variable kmer sizes $(-\mathrm{k}=$ $21,33,55,77,99$, or 127). The resulting contigs were fed into Prokka [67] for ORF calling, gene annotation, and generation of protein sequence files. Prokka-annotated protein sequences were used as input to mebipred.

\section{Results and Discussion.}

Available metal-binding protein structures are not diverse. The high resolution ${ }_{29}$ structure of most proteins is yet unknown, although this may change soon [68]. If a 
protein is of particular interest for the scientific community, it might be overrepresented in the PDB; e.g. over 1,300 structures of the SARS-COV2 spike sprotein. Thus, whether the known protein structures are a representative sample of all naturally occurring proteins is debatable and outside the scope of this work [69]. ${ }_{5}$ However, available structures constitute the most reliable set of metal-binding sproteins [70, 71]. A quarter $(49,996$ of 152,346$)$ of the PDB entries contain at least 7one of the metal atoms considered here. However, removing redundancy (at $70 \%$ ssequence identity using CD-HIT [59]) retains 39,066 structures, of which only $9 \%$ are metal-binding (3,542 metal binding; note that a single sequence can bind multiple 10different ligands; SOM Data:PDB_chain_<METAL>_5.0A); the number of structures binding each ion varies (SOM Table 1),

mebipred attains exemplary performance. In cross-validation, the first (binary; 1зyes/no) layer of mebipred identified sequence non-redundant metal-binding proteins 4 with nearly $80 \%$ precision at a $50 \%$ recall $\left(F 1_{\text {max }}=0.72\right.$ defines the default cutoff $=0.4$; ${ }_{5}$ Eqn. 1) - almost twice the precision obtained by BLAST at a similar recall (Fig. 3A); sperformance on the set of proteins including redundant sequences was, as expected, 7even higher $\left(F 1_{\max }=0.83\right.$ at default cutoff $)$.

${ }_{18}$ Note that performing the BLAST search for all sequences in the PDB took 19approximately six weeks (for 500,411 chain sequences in 152,346 structures), on 20average 7.25 seconds/sequence on one core of a $2.4 \mathrm{GHz}$ machine with 16G 1 RAM). The same dataset was processed by mebipred on the same machine in 29 ${ }_{22}$ minutes $\left(\sim 6.3^{*} 10^{-5}\right.$ seconds/sequence). While both BLAST and mebipred can be run 23 with multiple cores, the difference in speed is likely to be retained. Moreover, BLAST ${ }_{24}$ compute time is expected to grow both with database size and the number of queries ${ }_{25}[72]$, while mebipred prediction time only reflects the number of queries, i.e. the 26algorithm scales as $(O) n$.

\section{$<<$ FIG 3>>>}

28The second layer of mebipred predicts binding specifically to a ligand containing one 29 of the ten ions under consideration. In cross-validation using our data set (Methods), 
Imebipred was accurate in predicting ion specificity of individual proteins (Table 1). ${ }_{2}$ Note that we did not build predictors for proteins binding other biologically active smetals (e.g. vanadium, molybdenum, titanium, etc.), because the number of 4 structures binding these was insufficient to train a model of this kind. These could be sincorporated into mebipred in the future if more protein structures binding these ${ }_{6}$ metals are resolved.

Table 1: mebipred performance across metals

\begin{tabular}{|l|l|l|l|l|l|l|l|l|l|l|l|l|}
\hline ANN & $\begin{array}{l}\text { Metal- } \\
\text { binding }\end{array}$ & $\mathrm{Fe}$ & $\mathrm{Ca}$ & $\mathrm{Na}$ & $\mathrm{K}$ & $\mathrm{Mg}$ & $\mathrm{Mn}$ & $\mathrm{Cu}$ & $\mathrm{K}$ & $\mathrm{Co}$ & $\mathrm{Ni}$ & $\mathrm{Zn}$ \\
\hline AUC & 0.91 & 0.95 & 0.86 & 0.83 & 0.91 & 0.82 & 0.91 & 0.97 & 0.91 & 0.85 & 0.91 & 0.90 \\
Precision* & 0.80 & 0.96 & 0.91 & 0.86 & 0.88 & 0.79 & 0.89 & 0.98 & 0.88 & 0.89 & 0.84 & 0.95 \\
Recall* & 0.50 & 0.91 & 0.77 & 0.68 & 0.84 & 0.80 & 0.83 & 0.92 & 0.84 & 0.71 & 0.67 & 0.70 \\
F1 measure* & 0.71 & 0.94 & 0.83 & 0.76 & 0.86 & 0.80 & 0.86 & 0.95 & 0.86 & 0.79 & 0.75 & 0.80 \\
\hline
\end{tabular}

$8^{*} \mathrm{~F} 1$ measure, Precision, and Recall are reported at a default cutoff $=0.4$ for the first layer, which predicts metal gbinding, and at default cutoff=0.5 (In both cases established via maximum value of $F 1$ measure, $F 1_{\max }$ ) for second lolayer of per ion mebipred predictions. Note that at a cutoff $=0.5$, the first layer attains 0.86 precision at 0.24 recall.

Note that the predictions of the second layer of mebipred do not always match those in of the first layer. A generic metal binding prediction can still be true in the absence of sthe specific ion binding prediction; i.e. a protein can bind metals that are not part of our ion collection, e.g. Titanium, Vanadium, etc. A different type of discrepancy is 15when the first layer predicts the protein to not be able to bind metals, while the 16 second identifies a specific ion preference. We evaluated the second layer's ability to 1. predict metal binding by considering any positive (at the default cutoff $=0.5$ for each 1sion) ion binding prediction as indication of metal binding. This approach has a precision of 0.38 and a recall of 0.8 ; increasing stringency to cutoff of 0.9 , improves zoperformance (precision=0.8, recall=0.78). For comparison, the first layer at the default cutoff of 0.4 , has the same precision and a lower recall of 0.5 (Table 1 ). ${ }_{22}$ These observations suggest that in cases of disagreement between the layers, high 23scoring predictions of the second layer can be trusted to guide overall metal binding 24 predictions. 
Our evaluation of mebipred performance against that of other methods on our data was complicated by the absence of available web servers/standalone packages. 3 Thus, we ran our tool on the data reported by the different methods . mebipred spredicted metal-ligand binding better than Metal Detector2 [50] (Table 2) - a tool 5designed to predict transition metal-binding sites. Our method was also better than MetalExplorer [37], which predicts binding of eight metal ions with a precision of $60 \%$ 7 at a range of recalls from $59 \%$ to $88 \%$; mebipred attained an average of $80 \%$ sprecision for the same recall range. It also outperformed [40] (Table 3) and [46] 9 (Table 3) methods, but performed worse than structure-based MIB [23]. Note that tohere we used the measure of accuracy (Eqn. 1) since it was reported in the corresponding publications, but precision and recall might be more relevant for 12imbalanced datasets [73].

Table 2: mebipred performance vs. MetalDetector2

\begin{tabular}{|c|c|c|c|c|}
\cline { 3 - 5 } \multicolumn{2}{c|}{} & \multicolumn{2}{c|}{ Precision(\%) } & Recall(\%) \\
\hline Ligand & N & MetalDetector2 & mebipred & \\
\hline Zn & 817 & 63 & 90 & 70 \\
\hline Fe (Heme)* & 234 & 67 & 93 & 77 \\
\hline Fe(Fe- S)* & 202 & 68 & 97 & 67 \\
\hline $\mathrm{Cu}$ & 87 & 57 & 96 & 64 \\
\hline
\end{tabular}

$14^{*}$ Note that for Heme and Fe-S we report performance separately even though both methods predict only Fe 15binding.

Table 3 mebipred performance vs. other methods

\begin{tabular}{|c|c|c|c|c|}
\hline \multirow{2}{*}{ Ligand } & $\begin{array}{c}\text { Cao et al } \\
{[40]} \\
(\text { Acc\%) }\end{array}$ & $\begin{array}{c}\text { Kumar et al } \\
{[46](\text { Acc\%) }}\end{array}$ & $\begin{array}{c}\text { MIB [23] } \\
(\text { Acc\%) }\end{array}$ & $\begin{array}{c}\text { mebipred } \\
(\text { Acc\%) }\end{array}$ \\
\cline { 2 - 5 } & Sequence & Sequence & Structure & Sequence \\
\hline $\mathrm{Ca}$ & 74.8 & 75.4 & 94.1 & 86.7 \\
\hline $\mathrm{Co}$ & 83 & 85.3 & 94.7 & 86.2 \\
\hline $\mathrm{Cu}$ & 96.3 & 78.1 & 95.3 & 87.2 \\
\hline $\mathrm{Fe} 2$ & 91.3 & 75.6 & 95.1 & 89.2 \\
\hline $\mathrm{Fe} 3$ & 87.8 & 74 & 94.9 & 89.2 \\
\hline $\mathrm{K}$ & 80.3 & - & - & 74.0 \\
\hline $\mathrm{Mg}$ & 75.3 & 74 & 94.6 & 75.6 \\
\hline
\end{tabular}




\begin{tabular}{|c|c|c|c|c|}
\hline $\mathrm{Mn}$ & 83.2 & 68.8 & 95.0 & 89.7 \\
\hline $\mathrm{Na}$ & 79.4 & 79.4 & - & 84.5 \\
\hline $\mathrm{Ni}$ & - & 90.7 & 94.7 & 79.2 \\
\hline $\mathrm{Zn}$ & 83 & 69 & 94.8 & 82.2 \\
\hline
\end{tabular}

mebipred predicts protein metal-binding propensity from short fragments. We sextracted a set of 101,054,024 50-residue peptides from the PDB protein sequences 4 (Methods); these correspond to the typical lengths of peptides that could be sgenerated by translating DNA reads produced by next-gen sequencing [74]. We spredicted metal-binding for these fragments using mebipred and aligned them (via BLAST) to PDB sequences following the same procedure as for complete proteins (Methods; excluding hits to proteins from which fragments were produced). mebipred outperformed BLAST (Fig 3B) in identifying peptides generated from metal-binding oproteins. BLAST is not designed to deal with short sequence alignments $[61,75]$ and our results suggest that sequence identity may not be an accurate indicator of metal12binding either. Note that it is still possible that other alignment methods or changes in 13substitution matrices, i.e. penalizing the substitutions involving the residues often involved in metal binding, could yield better results.

Ion binding preferences are consistent per Pfam family. We ran mebipred on the 16607,903 Pfam proteins (8,207 families) whose structures are available in the PDB. ${ }_{17}$ According to binary predictions of the first layer, for $61 \%$ of the families, either all ımember proteins were predicted to be metal-binding or none were (SOM Data 19stats_with_id). Furthermore, for $\sim 41 \%$ of the families, either all member proteins were zopredicted to bind a given metal or none were (SOM Data: stats_with_id). Of predictions per metal, $69 \%$ were cases where no members of one family bind that 22 metal and $5 \%$ were cases where all of members of one family bind it - a total of $74 \%$ 23agreement of per ion predictions for members in the same family; the remaining $26 \%$ 24 of the family members were predicted to have different ion preferences (SOM Data: 25stats_with_id ). Our results indicate that metal binding preferences are mostly ${ }_{26}$ consistent within a Pfam family. This is expected, as Pfam domains reflect homology 
that, in turn, often suggests similar functionality and ligand binding [76, 77]. However, different ion preferences for a quarter of the families also suggest that specific metal зavailability within individual environments may have driven divergent evolution of new ligand-binding functionalities across organisms [22, 78]. Note that prediction error sand cambialistic activity (i.e. ability to bind multiple ions) of certain proteins, which is not captured by this summary of ion binding, could also contribute to this discrepancy in metal binding preferences of single family members.

\section{Mebipred predictions do not always reflect existing annotations of metal-} binding. We compared our METAL and NO_METAL datasets with Swiss-Prot metallobinding annotations. Of the 253,377 PDB sequences mapped to Swiss-Prot ${ }_{11}$ (PDBSWS [79]; April 2021), 53,652 ( 20\%) had annotations that disagreed with our data. Of these 32,667 were in our METAL set, i.e. in a PDB structure with a metal ion 13within $5 \AA$ of the chain (Methods) but were not described as metal binding by Swiss${ }_{4}$ Prot. Manual examination of ten randomly chosen discrepancies, confirms that the ${ }_{15}$ metal ion is present in a functional pocket, suggesting that Swiss-Prot annotations 16 are incomplete. The remaining 20,985 sequences were described in Swiss-Prot as 7 metal-binding but were not in our METAL set.

${ }_{18}$ We ran mebipred on these 20,850 PDB-Swiss-Prot discrepancies. Our predictions ${ }_{19}$ (binary metal binding at default cutoff) agreed with Swiss-Prot annotations two thirds 20 of the time $(64 \%, 13,374$ sequences, predicted metal-binding) sequences and with ${ }_{21}$ PDB otherwise (36\%, 7,476 sequences, predicted metal non-binding). Crystal 22 structures of metal-binding sequences may not contain a metal for a number of 23reasons, including biologically irrelevant binding (i.e. a metal can be bound by a 24 protein, but isn't under physiological conditions [80]) or experimental/technical ${ }_{25}$ crystallization decisions [16]. However, we expect that the $1,302(6 \%$ of 20,850$)$ non ${ }_{26}$ metal-binding chains from metal ion-containing PDB structures are most likely to be ${ }_{27}$ true non-binders of that ion. In fact, mebipred predictions for these proteins agreed 28 with PDB $41 \%$ of the time (540 sequences predicted to be nonbinding) - a somewhat 29 better agreement (vs 36\%) than that for other designated metal non-binders. 
A closer inspection further informs the reasons for database annotation differences. 2For example, 32 of the 540 predicted metal non-binding PDB chains map to the ${ }_{3}$ Rieske subunit of cytochrome BC1 - a Fe-S cluster binding protein (Swiss-Prot ID: ${ }_{4}$ Q5ZLR5) [81]. None of these 32 chains, however, are complete sequences of the sprotein and none contain the part of the structure that would bind the Fe-S cluster. In this particular case, the annotation discrepancy arises from a technical decision not to determine the metal-binding regions via crystallograhy [81]. While this level of sscrutiny for every disagreement between databases is beyond the scope of this work, 'we note that an annotation discrepancy doesn't necessarily constitute a "bug" but, 1orather, a feature of the method; i.e. mebipred could be used to resolve annotation conflicts between databases.

Mebipred can predict metal-binding from metagenome read translations. We compared the metal-binding profiles of the Black Sea metagenomic (assembled and inot assembled) samples obtained at different depths in a water column [82] (six ssamples (SRR12347146, SRR12347144, SRR12347141, SRR12347140, 6SRR12347142, SRR12347143, extracted from NCBI-SRA DB [83] and processed as 17in Methods). The relative frequencies of the resulting metal-binding protein/peptide spredictions were very similar (Table 4; Euclidean distance between metagenome samples ( $p$ and $q$; Eqn. 2) $=0$, where $n \in(\mathrm{Ca}, \mathrm{Co}, \mathrm{Cu}, \mathrm{Fe}, \mathrm{K}, \mathrm{Mg}, \mathrm{Mn}, \mathrm{Na}, \mathrm{Ni}, \mathrm{Zn}$ ), zoindicates identical metal-binding frequency profiles).

$$
\text { Euclidean Distance }(p, q)=\sqrt{\left(p_{1}-q_{1}\right)^{2}+\left(p_{2}-q_{2}\right)^{2}+\cdots+\left(p_{10}-q_{10}\right)^{2}} \text { (Eqn. 2) }
$$

22 This result suggests that mebipred can reliably predict metal-binding from 23translations of metagenomic reads (Methods).

\section{Table 4: Relative frequencies of metal binding proteins in metagenomic ssamples.}

\begin{tabular}{|c|c|c|c|c|c|c|c|c|c|c|}
\hline Assembled & & & & & $\%$ & & & & & \\
\hline SAMPLE (m) & $\mathrm{Ca}$ & Co & $\mathrm{Cu}$ & $\mathrm{Fe}$ & $\mathrm{K}$ & $\mathrm{Mg}$ & $\mathrm{Mn}$ & $\mathrm{Na}$ & $\mathrm{Ni}$ & $\begin{array}{c}\text { Euclidean } \\
\text { distance }\end{array}$ \\
\hline 50 & 19.32 & 2.57 & 4.68 & 15.37 & 7.79 & 21.38 & 7.62 & 10.9 & 4.35 & 0.66 \\
\hline 80 & 17.42 & 2.94 & 5.09 & 15.02 & 8.63 & 20.63 & 8.44 & 10.76 & 5.63 & 0.67 \\
\hline
\end{tabular}




\begin{tabular}{c|cccccccccc}
170 & 17.9 & 2.96 & 5.26 & 15.12 & 8.72 & 20.69 & 8.12 & 9.83 & 5.92 & 0.63 \\
250 & 17.67 & 2.91 & 5.23 & 15.97 & 8.18 & 20.38 & 8.06 & 10.32 & 5.42 & 0.65 \\
500 & 16.87 & 3.07 & 5.11 & 15.92 & 8.23 & 20.41 & 8.01 & 10.75 & 5.41 & 0.41 \\
1000 & 16.91 & 2.95 & 5.11 & 16.4 & 8.28 & 20.37 & 7.96 & 10.51 & 5.4 & 0.47 \\
2000 & 17.01 & 2.93 & 5.21 & 16.03 & 8.06 & 20.49 & 8.01 & 11.22 & 5.04 & 0.68
\end{tabular}

\begin{tabular}{|c|c|c|c|c|c|c|c|c|c|c|}
\hline Unassembled & & & & & $\%$ & & & & & \\
\hline SAMPLE (m) & $\mathrm{Ca}$ & Co & $\mathrm{Cu}$ & $\mathrm{Fe}$ & K & $\mathrm{Mg}$ & $\mathrm{Mn}$ & $\mathrm{Na}$ & $\mathrm{Ni}$ & $\begin{array}{c}\text { Euclidean } \\
\text { distance }\end{array}$ \\
\hline 50 & 19.04 & 2.60 & 4.70 & 15.60 & 7.59 & 20.89 & 7.65 & 10.77 & 4.24 & 0.66 \\
\hline 80 & 17.50 & 2.92 & 5.04 & 14.93 & 8.78 & 21.17 & 8.29 & 10.44 & 5.68 & 0.67 \\
\hline 170 & 18.24 & 2.94 & 5.32 & 15.41 & 8.83 & 20.97 & 7.98 & 10.12 & 5.91 & 0.63 \\
\hline 250 & 17.63 & 2.83 & 5.24 & 15.89 & 8.07 & 19.82 & 8.24 & 10.10 & 5.37 & 0.65 \\
\hline 500 & 16.81 & 3.08 & 5.15 & 15.86 & 8.01 & 20.16 & 8.15 & 10.67 & 5.25 & 0.41 \\
\hline 1000 & 17.18 & 2.99 & 5.05 & 16.69 & 8.10 & 20.41 & 8.00 & 10.38 & 5.47 & 0.47 \\
\hline 2000 & 17.23 & 2.97 & 5.11 & 15.70 & 7.91 & 21.01 & 7.95 & 11.16 & 5.01 & 0.68 \\
\hline
\end{tabular}

Diversity of metal-binding proteins highlights environmental differences. ${ }_{3}$ Across a number of environmental samples, we observed protein metal-binding usignatures consistent with environmental features and subtypes.

5 Black Sea water column: From the above analysis we observed that the percentage of reads predicted as metal binding was approximately $1 \%$ for all Black Sea samples 7(SOM Table 4). The Black Sea is a heavily stratified body of water, where $\mathrm{pH}$, soxygen, and light gradients have been characterized [84]. The sea surface layers where photosynthesis can occur, i.e. the epipelagic zone, are, by definition, up to $10200 \mathrm{~m}$ in depth; on the Black Sea, however, almost no photosynthetic activity can be found below 100m [85]. The epipelagic zone samples in our set are slightly enriched ${ }_{12}(2 \%$ increase) in Mg-binding proteins (Fig. 4). This is in line with the use of $\mathrm{Mg}$ in schlorophyll [86].

$$
<<\text { FIG 4>>> }
$$

IIIn non-photosynthetic environments, we observed a trade-off between the 16enrichment of $\mathrm{Mg}$ and Fe binding proteins, which can be accounted for by the lower ${ }_{17} \mathrm{pH}$ increasing $\mathrm{Fe}$ availability and by the abundance of iron-reducing organisms at 18greater depths [87, 88]. The maximal difference between the abundances of 
predicted metal-binding proteins is observed between the samples taken at 50 and 2170 meters, i.e. bypassing the photosynthetic limit; as indicated by the steep slope of the line tracing the Euclidian distance between metal-binding protein abundance 4 vectors of individual samples (Fig. 4). Sample metal-binding preferences appear smore similar below 170m (lower absolute value of slope). The difference between consecutive depths until $1,000 \mathrm{~m}$ is in line with the changes in the environment 7 described by the $\mathrm{pH}$ chemocline, changes in reduction potential, and reduced light [89]; i.e. the deeper one goes the lower the $\mathrm{pH}$, the less calcium, and the more $\mathrm{Fe}$ [90]. The change in the sign of the slope indicating increasingly different samples at $101,000 \mathrm{~m}$ and 2,000m likely accompanies a change in the microbial community [82]. 11 This may reflect the transition from the Mesopelagic $(200 \mathrm{~m}$ to $1000 \mathrm{~m})$, where some 12light and oxygen are still available, to the Antropelagic region (1000m to 4000m), 13where there isn't any of either. Alternatively, this change can highlight the fact that $42000 \mathrm{~m}$ is essentially the seafloor [91].

${ }_{5}$ Hot spring sediments: To test mebipred we analyzed 16 metagenomic samples from 16hot spring sediments obtained from NCBI-SRA DB (accession numbers 7SRS6512487, SRS6512485, SRS6512489, SRS6512493, SRS6512459, SRS6512462, SRS6512461, SRS6512467, SRS6512468, SRS6512471, SRS6512470, SRS6512473, SRS6512475, SRS6512481, SRS6512479, SRS6512483) and previously described in Fullerton et al. [92]. The proportion of ${ }_{21}$ genetically encoded proteins binding each metal was similar (within $2 \%$ ) for all 22samples (SOM Table 2). We observed a significant correlation between the relative 23 frequency of proteins iron-binding iron and the iron environmental concentrations ${ }_{24}($ Pearson $r=0.54$ p-val = 0.03; SOM Table 3); for zinc and manganese, the correlation ${ }_{25}$ was positive, but weak (Pearson $r=0.1$ and $0.18, p$-val $=0.71$ and 0.05 , ${ }_{26}$ respectively). Copper and nickel binding proteins, on the other hand, had a negative ${ }_{27}$ correlation (but not significant) with the corresponding environmental concentrations ${ }_{28}($ Pearson $r=-0.1 / p-v a l=0.7$ and Pearson $r=-0.43 / p-v a l=0.1$, respectively)

${ }_{2}$ We lack complete information about metal requirements for different microbial 30 strains. However, there is evidence that metabolites reflect the microbial community 
composition by altering the abundance of metabolite-relevant genes [93] - a finding somewhat in line with our observations. However, why did only iron (Fe) concentrations significantly correlate with iron-binding protein abundance? $\mathrm{Fe}$ is 4 considered a major element (>1,000ppm), while others $(\mathrm{Zn}, \mathrm{Mn}, \mathrm{Cu}, \mathrm{Ni})$ are trace selements (<100 ppm) [94-96]. Metabolic requirements for each metal vary across organisms. However, iron is essential for nearly all of them; e.g., restricting iron 7availability to microbial invaders is part of the innate immune response [97]. ${ }_{8}$ Additionally, of the five measured metals, $\mathrm{Fe}$ is the only one that is present in the sampling sites at concentrations (observed: 3 - 400 ppm) below the what is needed Ifor growth of metal requirement annotated bacteria [94, 96] (average requirement: 5,400 ppm); in fact, bacteria aim to actively accumulate Fe using specialized proteins [98]. The other four metals are usually required in concentrations [96] below those sobserved in this study. Moreover, higher concentrations may be deleterious to organism fitness, particularly for the anticorrelated metals. For example, nickel is ${ }_{15}$ required in trace quantities [99] and competes with $\mathrm{Mg}$ and $\mathrm{Ca}$ for binding sites [100]; 16in high concentrations, it can also damage DNA [101]. Copper is frequently toxic for bacteria at environmental concentrations [102] and is thus tightly regulated. Thus, given its key role in metabolism and limiting factor status, iron concentrations could drive microbial selection and explain the abundance of genes encoding iron binding proteins.

Human-host microbiomes: We further used mebipred to analyse randomly chosen 22human host and soil microbiome samples from the NCBI-SRA DB (SRR13422487, 3SRR12347145, ERR2855085, ERR5056238， SRR13422469， SRR12347144, SRR12432127， SRR12347141，SRR12360453，SRR12347140， SRR12347142, 5ERR2855082， SRR12347143， SRR12432116, SRR12347146, ERR2728283). ${ }_{6}$ Predicted metal-binding proteins (Fig. 5) are in line with the available metals in each environment. For example, few or no iron-binding proteins are predicted in samples of human origin except for one vaginal sample, where the occurrence may be 29explained by menstrual cycle bleeding. Low concentrations of iron-binding proteins 3oare observed in the gut and pregnancy-associated vaginal microbiota, both of which 
Imay be accounted for by minor bleed episodes. As mentioned above, iron sequestering is part of normal human immune response and is lethal to most зpathogenic bacteria [97]; normal non-pathogenic microbiota are likely to be adapted to low iron environment [103].

$$
<<<F \mid G 5>>
$$

Metal-binding proteins predicted to occur in the soil and in gut samples target more 7different metals than do skin, mouth, and vaginal samples, likely due to the metabolic diversity of the former [104, 105]. The predicted metal-binding proteins in skin samples target metals ( $\mathrm{Ca}, \mathrm{K}, \mathrm{Mg}, \mathrm{Mn}$ ) that can be found in sweat in relatively high ioconcentrations (>1mg/l) [106]. Other metals (e.g. Zn, Cu) are present in sweat in 11 trace concentrations $(<1 \mathrm{mg} / \mathrm{l})$ [107, 108] and, consequently, few proteins bindings these metals are predicted ( $<1 \%$ of predictions). Furthermore, the differences in smetal-binding protein abundances between vaginal samples from pregnant and nonispregnant women could reflect the large changes in the vaginal microbiome 15associated with pregnancy [109].

${ }_{16}$ mebipred is an advance in the field of function prediction from protein sequence, 17which we showed to be applicable to the annotation of metagenomic samples. It can 18help resolve database annotation errors and shows potential for linking function with environmental conditions. We further expect that as more metal-binding protein 20structures are resolved, our method can be improved and expanded, for example to 21the detection of to other metals not currently treated. Its capacity to annotate metal 22binding informs the descriptions of microbiome environmental conditions and ${ }_{3}$ diversity. Finally, since most enzymes are metal binding proteins, it could also help 24enzyme prospecting in future studies.

\section{${ }_{5}$ Conclusion}

26 Here we compiled a gold-standard experimentally-derived metal-binding protein set 27and built mebipred - a sequence-based neural network predictor of metal binding. 28 mebipred significantly outperforms existing sequence-based methods for annotation 29of metal binding; it also detects specific metals bound by each protein. We expect 
that the growth in the number of metal binding proteins with resolved structures will make these types of approaches even more powerful in the near future. To the best sof our knowledge, mebipred is also the only reference-free sequence-based tool for identifying metal-binding. Our method is faster than existing tools and can predict 5 metal binding using short protein fragments - both characteristics that make it useful in analysis of metagenomic data. In evaluation of microbiome samples we found that 7 differences in the number of predicted metal-binding proteins were related to the sconcentration of metal ions in the corresponding environments.

\section{Acknowledgments}

${ }_{11}$ We are grateful to Drs. Paul Falkowski, Maximilian Miller, Chengsheng Zhu, Yannick ${ }_{12}$ Mahlich, Kenneth McGuinnes, Adrienne Hoarfrost, Natalia Rigazio, and Zishuo Zeng 13(all Rutgers) for their critical comments on the development of the method. We would 14also like to express gratitude to the PDB team and to all researchers that solve and ${ }_{15}$ deposit protein structures into the PDB for nearly half a century. Without them this 16work, and most of other structural bioinformatics work, would not be possible.

${ }_{17} \mathrm{DG}$ has received funding from the European Research Council (ERC) under the 18European Union's Horizon 2020 research and innovation programme (Grant 19agreement No. 948972) ERC-STG-2020 project CoEvolve. DUF is a fellow of the ${ }_{20}$ CONICET. Y.B. and A.A. were supported by the NASA Astrobiology Institute grant 2180NSSC18M0093. Y.B. was also supported by the NSF (National Science ${ }_{22}$ Foundation) CAREER award 1553289. 


\section{References}

21. Arnold, F.H. and J.-H. Zhang, Metal-mediated protein stabilization. Trends in biotechnology, 1994. 12(5): p. 189-192.

42. Slater, J.P., A.S. Mildvan, and L.A. Loeb, Zinc in DNA polymerases. Biochemical and biophysical research communications, 1971. 44(1): p. 37-43.

63. Batra, V.K., et al., Magnesium-induced assembly of a complete DNA polymerase catalytic complex. Structure, 2006. 14(4): p. 757-766.

84. Yang, L., et al., Critical role of magnesium ions in DNA polymerase $\beta$ 's closing and active site assembly. Journal of the American Chemical Society, 2004. 126(27): p. 8441-8453.

15. Bennett, L.E., Metalloprotein redox reactions, in Current Research Topics in Bioinorganic Chemistry. 1973, John Wiley Springfield, III.

36. Falkowski, P.G., Life's Engines. 2015: Princeton University Press.

47. Moore, E.K., et al., Metal availability and the expanding network of microbial metabolisms in the Archaean eon. Nature Geoscience, 2017. 10(9): p. 629636.

78. Jelen, B.I., D. Giovannelli, and P.G. Falkowski, The role of microbial electron transfer in the coevolution of the biosphere and geosphere. Annual review of microbiology, 2016. 70: p. 45-62.

209. Bernstein, F.C., et al., The Protein Data Bank: A computer-based archival file for macromolecular structures. European journal of biochemistry, 1977. 80(2): p. 319-324.

10. UniProt: The universal protein knowledgebase in 2021. Nucleic Acids Research, 2021. 49(D1): p. D480-D489.

511. Consortium, U., UniProt: a worldwide hub of protein knowledge. Nucleic acids research, 2019. 47(D1): p. D506-D515.

2712. Whittaker, J.W., The irony of manganese superoxide dismutase. 2003, Portland Press Ltd.

2913. Deng, L., et al., Direct quantification of protein- metal ion affinities by electrospray ionization mass spectrometry. Analytical chemistry, 2010. 82(6): p. 2170-2174.

14. Handing, K.B., et al., Characterizing metal-binding sites in proteins with $X$-ray crystallography. Nature protocols, 2018. 13(5): p. 1062.

15. Lancaster, V.L., et al., A cambialistic superoxide dismutase in the thermophilic photosynthetic bacterium Chloroflexus aurantiacus. Journal of bacteriology, 2004. 186(11): p. 3408-3414.

16. Laganowsky, A., et al., An approach to crystallizing proteins by metal-mediated synthetic symmetrization. Protein Science, 2011. 20(11): p. 1876-1890.

17. Goto, J.J., et al., Loss of in vitro metal ion binding specificity in mutant copperzinc superoxide dismutases associated with familial amyotrophic lateral sclerosis. Journal of Biological Chemistry, 2000. 275(2): p. 1007-1014. 
18. Chaudhuri, B.N., et al., Structure of D-allose binding protein from Escherichia coli bound to $D$-allose at $1.8 \AA$ resolution. Journal of molecular biology, 1999 . 286(5): p. 1519-1531.

419. Lavecchia, A. and C. Di Giovanni, Virtual screening strategies in drug discovery: a critical review. Current medicinal chemistry, 2013. 20(23): p. 2839-2860.

720. Yamashita, M.M., et al., Where metal ions bind in proteins. Proceedings of the National Academy of Sciences, 1990. 87(15): p. 5648-5652.

21. Nayal, M. and E. Di Cera, Predicting Ca (2+)-binding sites in proteins. Proceedings of the National Academy of Sciences, 1994. 91(2): p. 817-821.

122. Un, S., et al., Manganese (II) zero-field interaction in cambialistic and manganese superoxide dismutases and its relationship to the structure of the metal binding site. Journal of the American Chemical Society, 2004. 126(9): p. 2720-2726.

23. Lin, Y.-F., et al., MIB: metal ion-binding site prediction and docking server. Journal of chemical information and modeling, 2016. 56(12): p. 2287-2291.

1724. Babor, M., et al., Prediction of transition metal-binding sites from apo protein structures. Proteins: Structure, Function, and Bioinformatics, 2008. 70(1): p. 208-217.

2025. Gregory, D.S., et al., The prediction and characterization of metal binding sites in proteins. Protein Engineering, Design and Selection, 1993. 6(1): p. 29-35.

226. Sodhi, J.S., et al., Predicting metal-binding site residues in low-resolution structural models. Journal of molecular biology, 2004. 342(1): p. 307-320.

2427. Levy, R., M. Edelman, and V. Sobolev, Prediction of 3D metal binding sites from translated gene sequences based on remote-homology templates. Proteins: Structure, Function, and Bioinformatics, 2009. 76(2): p. 365-374. Liu, T. and R.B. Altman, Prediction of calcium-binding sites by combining loopmodeling with machine learning. BMC structural biology, 2009. 9(1): p. 72. Zhao, W., et al., Structure-based de novo prediction of zinc-binding sites in proteins of unknown function. Bioinformatics, 2011. 27(9): p. 1262-1268.

\section{8.}

3431. Hecht, M., Y. Bromberg, and B. Rost, Better prediction of functional effects for sequence variants. BMC genomics, 2015. 16(8): p. 1-12.

3632. Koohi-Moghadam, M., et al., Predicting disease-associated mutation of metalbinding sites in proteins using a deep learning approach. Nature Machine Intelligence, 2019. 1(12): p. 561-567.

3933. Miller, M., et al., funtrp: identifying protein positions for variation driven functional tuning. Nucleic acids research, 2019. 47(21): p. e142-e142.

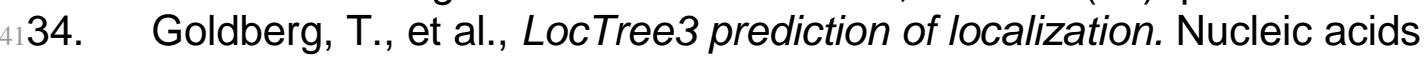
42 research, 2014. 42(W1): p. W350-W355.

4335. Lu, C.H., et al., The fragment transformation method to detect the protein structural motifs. Proteins: Structure, Function, and Bioinformatics, 2006. 63(3): p. 636-643. 
36. Lu, C.-H., et al., Prediction of metal ion-binding sites in proteins using the fragment transformation method. PloS one, 2012. 7(6).

37. Song, J., et al., MetalExplorer, a bioinformatics tool for the improved prediction of eight types of metal-binding sites using a random forest algorithm with twostep feature selection. Current Bioinformatics, 2017. 12(6): p. 480-489.

638. Mahlich, Y., et al., HFSP: high speed homology-driven function annotation of proteins. Bioinformatics, 2018. 34(13): p. i304-i312.

839. Capdevila, D.A., K.A. Edmonds, and D.P. Giedroc, Metallochaperones and metalloregulation in bacteria. Essays in biochemistry, 2017. 61(2): p. 177-200.

1040. Cao, X., et al., Identification of metal ion binding sites based on amino acid 11 sequences. PloS one, 2017. 12(8).

1241. Bateman, A., et al., The Pfam protein families database. Nucleic acids research, 2002. 30(1): p. 276-280.

42. Andreini, C., I. Bertini, and A. Rosato, $A$ hint to search for metalloproteins in gene banks. Bioinformatics, 2004. 20(9): p. 1373-1380.

43. Nakata, K., Prediction of zinc finger DNA binding protein. Bioinformatics, 1995. 11(2): p. 125-131.

44. Passerini, A., et al., Predicting zinc binding at the proteome level. BMC 19 bioinformatics, 2007. 8(1): p. 39.

2045. Passerini, A., et al., Identifying cysteines and histidines in transition-metalbinding sites using support vector machines and neural networks. Proteins: Structure, Function, and Bioinformatics, 2006. 65(2): p. 305-316.

346. Kumar, S., Prediction of metal ion binding sites in proteins from amino acid sequences by using simplified amino acid alphabets and random forest model. Genomics \& informatics, 2017. 15(4): p. 162.

647. Lin, C.-T., et al., Protein metal binding residue prediction based on neural networks. International journal of neural systems, 2005. 15(01n02): p. 71-84.

2848. Lippi, M., et al., MetalDetector: a web server for predicting metal-binding sites and disulfide bridges in proteins from sequence. Bioinformatics, 2008. 24(18): p. 2094-2095.

49. Ceroni, A., et al., DISULFIND: a disulfide bonding state and cysteine connectivity prediction server. Nucleic acids research, 2006.34(suppl_2): p. W177-W181.

450. Passerini, A., M. Lippi, and P. Frasconi, MetalDetector v2. 0: predicting the geometry of metal binding sites from protein sequence. Nucleic acids research, 2011.39(suppl_2): p. W288-W292.

51. Hamelryck, T. and B. Manderick, PDB file parser and structure class implemented in Python. Bioinformatics, 2003. 19(17): p. 2308-2310.

3952. Li, Z., J. Tang, and F. Guo, Identification of 14-3-3 proteins phosphopeptidebinding specificity using an affinity-based computational approach. PloS one, 2016. 11(2).

53. Jenks, G.F., The data model concept in statistical mapping. International yearbook of cartography, 1967. 7: p. 186-190.

54. Chollet, F., keras. 2015. 
55. Abadi, M., et al. Tensorflow: A system for large-scale machine learning. in 12th \{USENIX\} Symposium on Operating Systems Design and Implementation (\{OSDI\} 16). 2016.

456. Bengio, Y., Rmsprop and equilibrated adaptive learning rates for nonconvex optimization. corr abs/1502.04390, 2015.

657. Chollet, F., Introduction to keras. March 9th, 2018.

758. Huang, G.-B., Learning capability and storage capacity of two-hidden-layer feedforward networks. IEEE transactions on neural networks, 2003. 14(2): $p$. 274-281.

059. Fu, L., et al., CD-HIT: accelerated for clustering the next-generation sequencing data. Bioinformatics, 2012. 28(23): p. 3150-3152.

260. Camacho, C., et al., BLAST+: architecture and applications. BMC bioinformatics, 2009. 10(1): p. 1-9.

61. Altschul, S.F., et al., Basic local alignment search tool. Journal of molecular biology, 1990. 215(3): p. 403-410.

62. Yang, J., A. Roy, and Y. Zhang, BioLiP: a semi-manually curated database for biologically relevant ligand-protein interactions. Nucleic acids research, 2012. 41(D1): p. D1096-D1103.

63. Bolger, A.M., M. Lohse, and B. Usadel, Trimmomatic: a flexible trimmer for Illumina sequence data. Bioinformatics, 2014. 30(15): p. 2114-2120.

64. Ewing, B. and P. Green, Base-calling of automated sequencer traces using phred. II. Error probabilities. Genome research, 1998. 8(3): p. 186-194.

365. Cock, P.J., et al., Biopython: freely available Python tools for computational molecular biology and bioinformatics. Bioinformatics, 2009. 25(11): p. $1422-$ 1423.

2666. Nurk, S., et al., metaSPAdes: a new versatile metagenomic assembler. Genome research, 2017. 27(5): p. 824-834.

267. Seemann, T., Prokka: rapid prokaryotic genome annotation. Bioinformatics, 2014. 30(14): p. 2068-2069.

3068. Jumper, J., et al., Highly accurate protein structure prediction with AlphaFold. Nature, 2021: p. 1-11.

269. Jaroszewski, L., et al., Exploration of uncharted regions of the protein universe. PLoS Biol, 2009. 7(9): p. e1000205.

340. Andreini, C., et al., MetalPDB: a database of metal sites in biological macromolecular structures. Nucleic acids research, 2012. 41(D1): p. D312D319.

771. Putignano, V., et al., MetalPDB in 2018: a database of metal sites in biological macromolecular structures. Nucleic acids research, 2018. 46(D1): p. D459D464.

472. Kent, W.J., BLAT—the BLAST-like alignment tool. Genome research, 2002. 12(4): p. 656-664.

273. Ferri, C., J. Hernández-Orallo, and R. Modroiu, An experimental comparison of performance measures for classification. Pattern Recognition Letters, 2009. 30(1): p. 27-38. 
174. Jünemann, S., et al., Updating benchtop sequencing performance comparison. Nature biotechnology, 2013. 31(4): p. 294-296.

375. Campagna, D., et al., PASS: a program to align short sequences. Bioinformatics, 2009. 25(7): p. 967-968.

576. Sharma, D., et al., Bioinformatic exploration of metal-binding proteome of zoonotic pathogen Orientia tsutsugamushi. Frontiers in genetics, 2019. 10: p. 797.

877. Kauffman, C. and G. Karypis, LIBRUS: combined machine learning and homology information for sequence-based ligand-binding residue prediction. Bioinformatics, 2009. 25(23): p. 3099-3107.

178. Rausell, A., et al., Protein interactions and ligand binding: from protein subfamilies to functional specificity. Proceedings of the National Academy of Sciences, 2010. 107(5): p. 1995-2000.

479. Martin, A.C., Mapping PDB chains to UniProtKB entries. Bioinformatics, 2005. 21(23): p. 4297-4301.

68. Pidugu, L.S.M., et al., Crystal structures of human 3-hydroxyanthranilate 3, 4dioxygenase with native and non-native metals bound in the active site. Acta Crystallographica Section D: Structural Biology, 2017. 73(4): p. 340-348.

81. Zhang, Z., et al., Electron transfer by domain movement in cytochrome bc 1. Nature, 1998. 392(6677): p. 677-684.

82. Cabello-Yeves, P.J., et al., Microbiome of the Black Sea water column analyzed by genome centric metagenomics. bioRxiv, 2020.

2383. Leinonen, R., et al., The sequence read archive. Nucleic acids research, 2010. 39(suppl_1): p. D19-D21.

2584. Stanev, E.V., On the mechanisms of the Black Sea circulation. Earth-Science Reviews, 1990. 28(4): p. 285-319.

275. Callieri, C., et al., The mesopelagic anoxic Black Sea as an unexpected habitat for Synechococcus challenges our understanding of global "deep red fluorescence". The ISME journal, 2019. 13(7): p. 1676-1687.

366. Chu, S., The influence of the mineral composition of the medium on the growth of planktonic algae: part I. Methods and culture media. The Journal of Ecology, 1942: p. 284-325.

87. Fredrickson, J.K. and Y.A. Gorby, Environmental processes mediated by ironreducing bacteria. Current opinion in biotechnology, 1996. 7(3): p. 287-294.

388. Canfield, D.E., T.W. Lyons, and R. Raiswell, A model for iron deposition to euxinic Black Sea sediments. American Journal of Science, 1996. 296(7): p. 818-834.

889. Jørgensen, B.B., et al., Sulfide oxidation in the anoxic Black Sea chemocline. Deep Sea Research Part A. Oceanographic Research Papers, 1991. 38: p. S1083-S1103.

90. Lewis, B. and W. Landing, The biogeochemistry of manganese and iron in the Black Sea. Deep Sea Research Part A. Oceanographic Research Papers, 1991. 38: p. S773-S803.

491. Karatay, O., Neal Ascherson: Black Sea. Karadeniz Araştırmaları, 2007(13): p. 159-163. 
92. Fullerton, K.M., et al., Effect of tectonic processes on biosphere-geosphere feedbacks across a convergent margin. Nature Geoscience, 2021. 14(5): $p$. 301-306.

493. Mallick, H., et al., Predictive metabolomic profiling of microbial communities using amplicon or metagenomic sequences. Nature communications, 2019. 10(1): p. 1-11.

794. Scherer, P., H. Lippert, and G. Wolff, Composition of the major elements and trace elements of 10 methanogenic bacteria determined by inductively coupled plasma emission spectrometry. Biological trace element research, 1983. 5(3): p. 149-163.

95. Mertz, W., The essential trace elements. Science, 1981. 213(4514): p. 13321338.

396. Rouf, M., Spectrochemical analysis of inorganic elements in bacteria. Journal of Bacteriology, 1964. 88(6): p. 1545-1549.

597. Ganz, T., Iron in innate immunity: starve the invaders. Current opinion in immunology, 2009. 21(1): p. 63-67.

798. Braun, V. and K. Hantke, Recent insights into iron import by bacteria. Current opinion in chemical biology, 2011. 15(2): p. 328-334.

99. Chivers, P.T., Nickel recognition by bacterial importer proteins. Metallomics, 2015. 7(4): p. 590-595.

2100. Yang, J. and J. Black, Competitive binding of chromium, cobalt and nickel to serum proteins. Biomaterials, 1994. 15(4): p. 262-268.

101. Sunderman Jr, F.W., Mechanisms of nickel carcinogenesis. Scandinavian journal of work, environment \& health, 1989: p. 1-12.

5102. Dupont, C.L., G. Grass, and C. Rensing, Copper toxicity and the origin of bacterial resistance—new insights and applications. Metallomics, 2011. 3(11): p. 1109-1118.

103. Yilmaz, B. and H. Li, Gut microbiota and iron: the crucial actors in health and disease. Pharmaceuticals, 2018. 11(4): p. 98.

30104. Fierer, N., Embracing the unknown: disentangling the complexities of the soil microbiome. Nature Reviews Microbiology, 2017. 15(10): p. 579-590.

3105. Xu, Z. and R. Knight, Dietary effects on human gut microbiome diversity. British Journal of Nutrition, 2015. 113(S1): p. S1-S5.

4106. Robinson, S. and A.H. Robinson, Chemical composition of sweat. Physiological reviews, 1954. 34(2): p. 202-220.

36107. Emmett, E., The excretion of trace metals in human sweat. Annals of Clinical \& Laboratory Science, 1978. 8(4): p. 270-275.

108. Saraymen, R., E. Kilic, and S. Yazar, Sweat copper, zinc, iron, magnesium and chromium levels in national wrestler. Inonu Universitesi Tip Fakultesi Dergisi, 2004. 11(1): p. 7-10.

1109. Romero, R., et al., The composition and stability of the vaginal microbiota of normal pregnant women is different from that of non-pregnant women. Microbiome, 2014. 2(1): p. 1-19. 


\section{Figures}

\section{Fig.1}



Figure 1: Deriving the physicochemical features: example of "number of hydrogen bonds". In the toy sequence AYEAHWII, the "number of hydrogen bonds" feature can take values of 2, 3, or 4 at each amino acid. Translating each amino acid to its corresponding value and then to its category yields 23424322 and LMHLHMLL. Here we show how the property-based features composition, transitions (L-M case: Low to Medium and Medium to Low), and distribution (Low case) are calculated. These and the remaining physicochemical property features (transitions for $\mathrm{M}-\mathrm{H}$ and $\mathrm{L}-\mathrm{H}$ and distributions for Medium and High cases) are computed in the same manner. 
Fig. 2
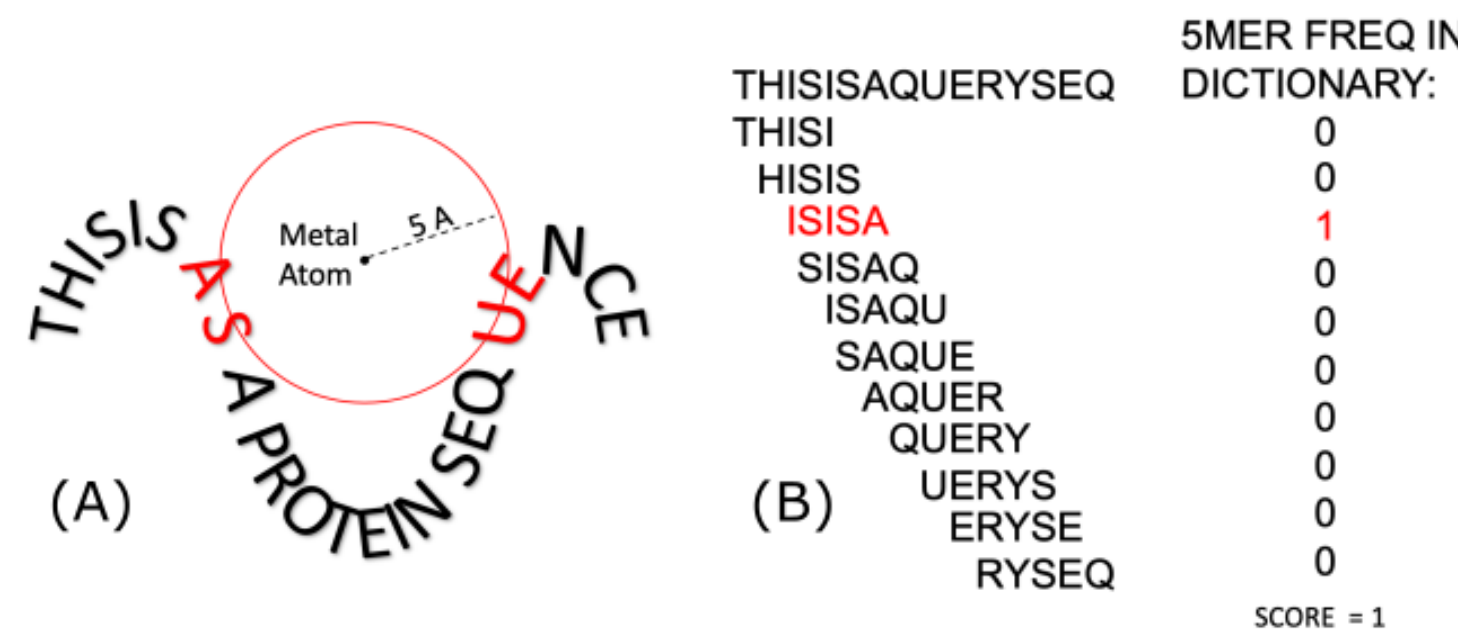

Figure 2: Deriving the 5 mer features. In this toy example of a protein structure that binds a metal, the red circle depicts a sphere of $5 \AA$ radius around the metal; the residues within the red circle are marked red. Each of these red residues and their neighboring residues (two on each side) make up a feature 5mer. In this example, the four 5mers are: "ISISA", "SISAP", "EQUEN" and "QUENC". The query sequence (right panel) is decomposed to count the number of metal-binding 5 mers present. The final score for this feature is the sum of the counts of all 5 mers in the sequence. 
Fig 3
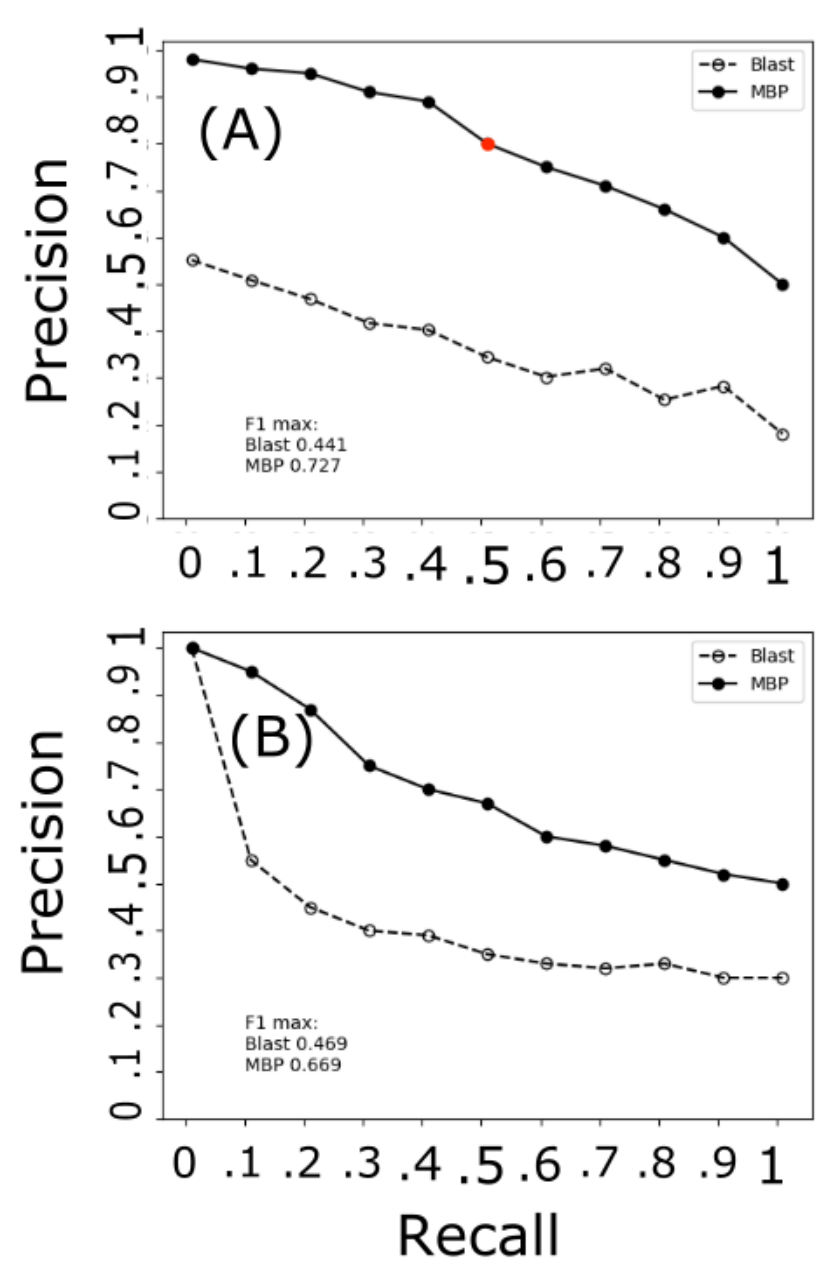

Figure 3. mebipred outperforms BLAST in identifying metal binding proteins and peptides. (A) At all cutoffs, mebipred (MBP; filled circles) is more precise than BLAST (empty circles). For example, at the default cutoff (score $=0.4$; red dot) it achieves $80 \%$ precision for half of the sequences ( $50 \%$ recall), as compared to $40 \%$ precision attained by BLAST. (B) mebipred also outperforms BLAST in identifying the metal binding propensity of proteins from their 50 amino acid fragment sequences. For example, for half of the fragments, it attains $67 \%$ accuracy, as compared to $39 \%$ attained by BLAST.

Fig 4 


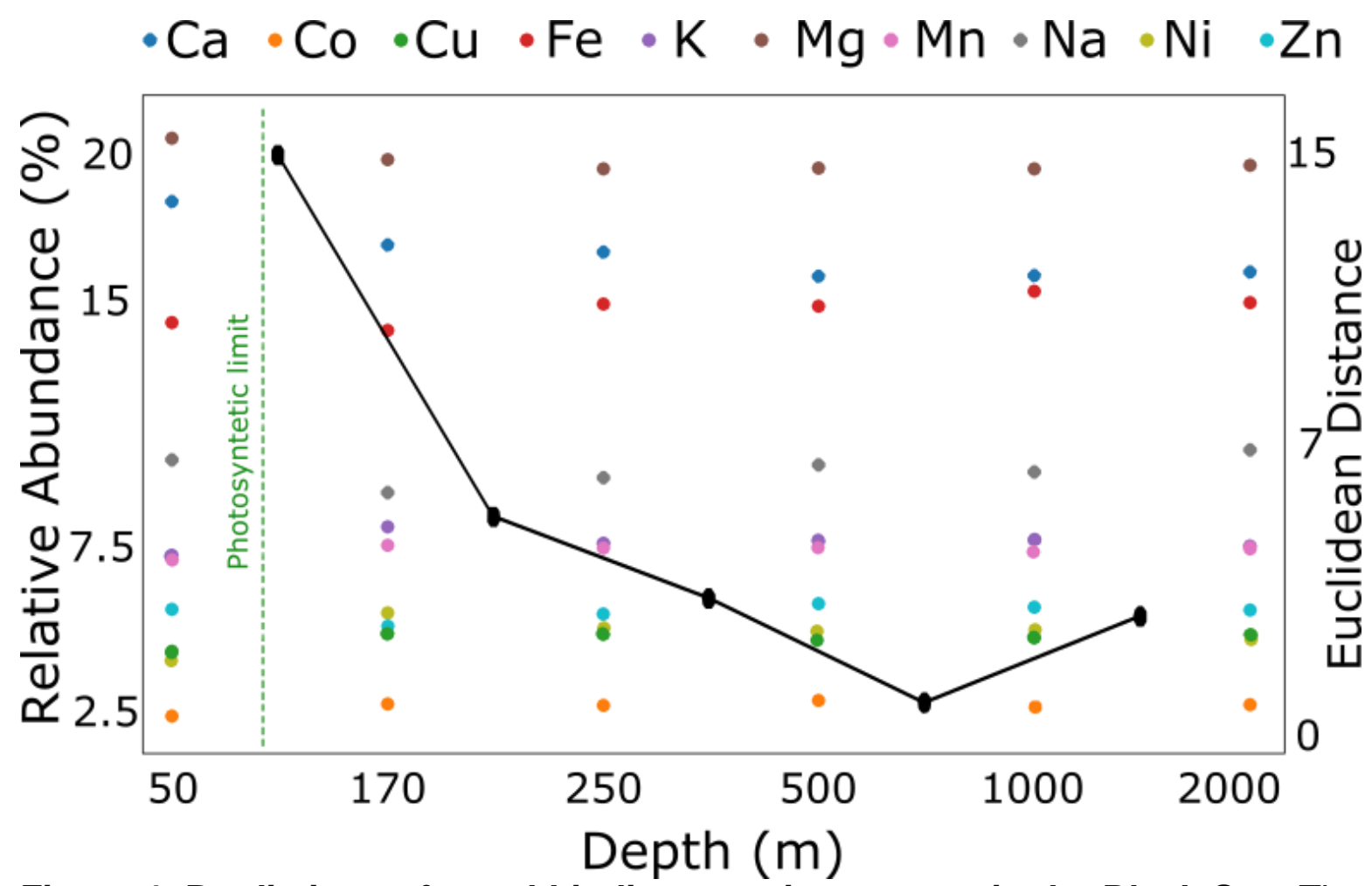

Figure 4. Predictions of metal binding proteins present in the Black Sea. The points on the graph indicate the relative abundance of ion-binding proteins (left $y$ axis) predicted from metagenomic samples collected at different depths of the Black Sea ( $x$ axis). The black line represents the Euclidean distance (right $y$-axis) between the vectors of predicted abundances at sequential depths. The markers on the line are placed between the depth measurements in each comparison. Samples show a phase transition (large Euclidean distance) at the photosynthetic limit (60 to 100m) \{Gorlenko, 2005 \#88;Callieri, 2019 \#89\}. 
Fig 5

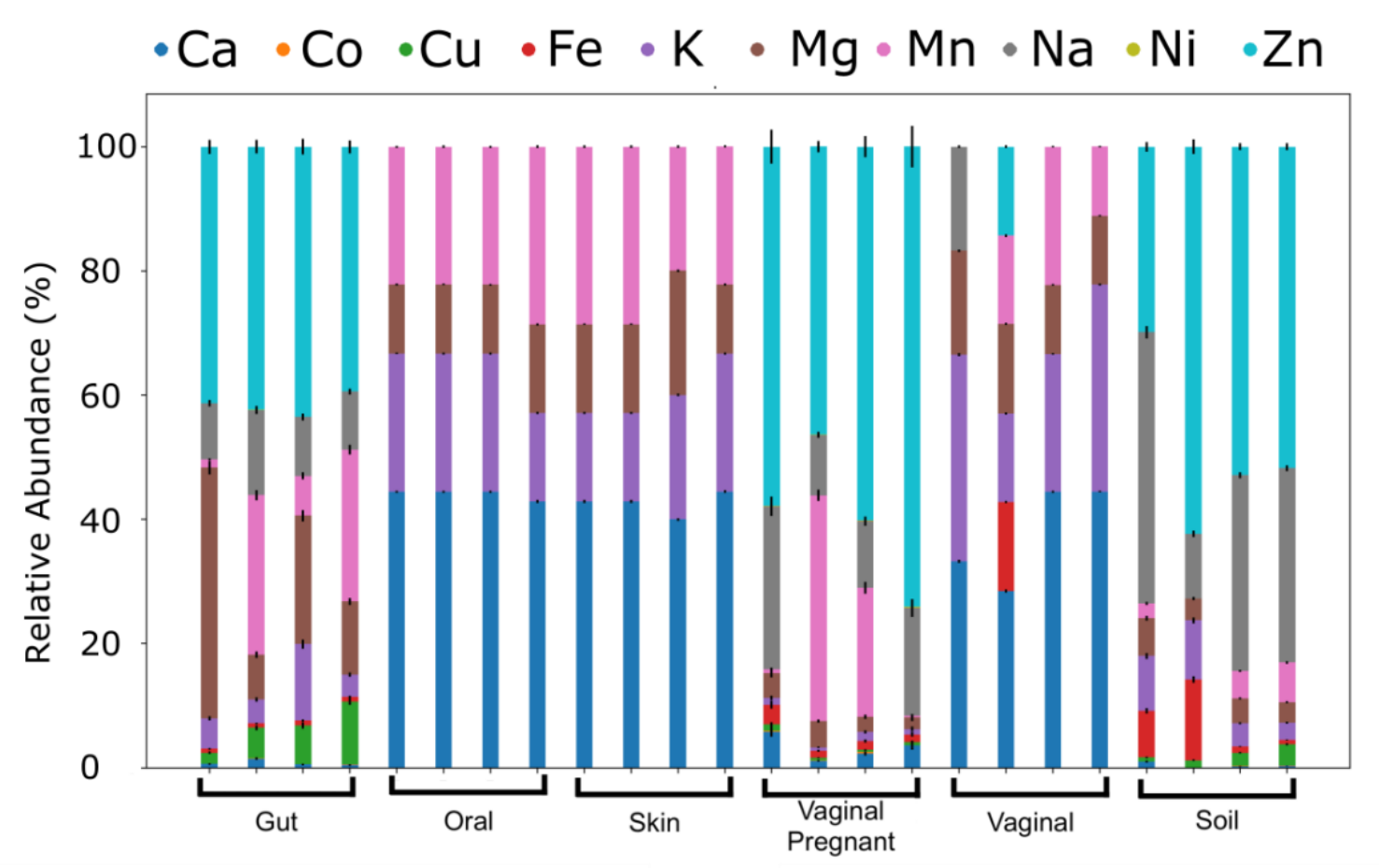

Figure 5: Differential abundance of metal binding proteins across environments. Each bar represents the relative abundance of predicted metalbinding proteins ( $y$-axis) in a given metagenomic sample; four sampled per environment (x-axis). Concentration of these proteins per environment (column colors and sizes) are similar within and different across environments, suggesting signature metal ion preferences. 